\title{
Un siècle d'aviation féminine : évasion, conquêtes et survol du continent africain
}

Jean-Marie Volet, University of Western Australia

En 1910, la baronne de Laroche obtenait le brevet no 36 de l'Aéroclub de France et devenait ainsi la première femme pilote au monde ${ }^{1}$. Quinze Françaises allaient suivre son exemple de 1910 à 1914. Madeleine Jaffeux, dont le baptême de l'air remonte à 1911, exprime bien l'attrait puissant que l'aviation exerçait sur un certain nombre de femmes de la Belle époque :

Les femmes font des rêves et souvent ceux-ci guident leur vie! Rêves de femmes! Chiffons, bijoux, autos ? Autre chose encore... J'ai rêvé comme mes semblables, mais j'ai longtemps fait un rêve plus beau... celui d'une grande traversée aérienne : quitter la France, escalader des montagnes, franchir la mer, voir d'autres pays, une autre civilisation, quitter la vieille Europe, manger du cassoulet le matin à Toulouse et le soir du couscous là-bas, au milieu des burnous, sous le ciel bleu d'Afrique qui vous arrache les yeux ! (Marck 133).

Alors que les pilotes s'élancent aux quatre points cardinaux, toujours plus haut, toujours plus loin, le rêve de Madeleine Jaffeux va rapidement devenir une réalité et les femmes rivalisent d'audace avec les hommes lors des premiers meetings aériens. Les records de hauteur et de distance tombent à un rythme effréné et il ne s'écoulera que quelques années avant que la Compagnie des Lignes Latécoère ne commence à assurer un acheminement régulier du courrier entre Toulouse et Casablanca (Chambe 239).

Nombre d'écrivains ont témoigné de cette conquête des cieux qui permit aux premiers voyageurs de se retrouver en Espagne, au Maroc, puis au cœur de l'Afrique en quelques coups d'ailes. Courrier Sud de Saint-Exupéry a donné ses lettres de noblesse à une avancée vers le sud qui atteindra rapidement les quatre coins du continent africain et le reste du monde, et l'on mentionne souvent les noms de Louis Blériot, Roland Garros, Guy Mermoz et bien d'autres pilotes entrés dans la légende. Bien qu'elles n'aient pas bénéficié de la même aura, les aviatrices ont réalisé des exploits non moins fascinants, car dès le début de l'aviation, de nombreuses femmes ont survolé la France, puis l'Afrique.

Plusieurs livres retracent leurs prouesses, de l'époque de Raymonde de Laroche à celle des Airbus, tout juste un siècle plus tard. Il est intéressant de découvrir par exemple que les premiers survols du sol africain remontent aux 
balbutiements de l'aviation. Raymonde de Laroche reçut en effet son brevet de pilote au terme d'un meeting aérien organisé au Caire en 1910. La pilote franchit ce jour-là une distance de vingt kilomètres, devenant du même coup la première femme à survoler le continent africain. Son «Voisin» était arrivé en Egypte par bateau, comme les avions des autres participants à ce meeting, car si l'on commençait à voler au nord comme au sud de la Méditerranée en 1910, il faudra attendre 1913 pour que Roland Garros, un jeune homme audacieux qui donnera son nom au stade de tennis le plus célèbre de France, ose se lancer dans la traversée de la mer séparant les deux continents. Parti de Saint-Raphaël le 23 septembre, il atterrit en Tunisie le même jour, sept heures cinquante-trois minutes après avoir décollé.

Certains fonctionnaires coloniaux français n'avaient cependant pas attendu cet exploit pour relever le potentiel civil et militaire de l'aviation. En 1910 déjà, le président de la Ligue Nationale Aérienne «sollicite le concours des troupes de terre stationnées dans le sud algérien en vue de faciliter les études préliminaires concernant l'organisation d'un service postal par aéroplanes dans le Sahara ( (Chambe 149) et la même année, le Gouverneur général de l'Afrique Occidentale Française écrit au Ministre des Colonies «pour lui signaler qu'en présence des résultats obtenus dans l'emploi des aéroplanes aux grandes manœuvres de 1910, il lui paraît avantageux de chercher à utiliser ce mode de locomotion dans certaines parties de l'A.O.F. [et propose] l'achat d'aéroplanes » (149). Tolérées dans les meetings aériens, les femmes ne furent cependant pas invitées à participer à ces premiers balbutiements de l'aviation coloniale. Elles ne furent pas davantage sollicitées comme aviatrices durant la guerre de 14/18 et les quelques femmes qui proposèrent de mettre sur pied une aviation sanitaire pour le transport des blessés, furent promptement remises à l'ordre par le Ministère de la guerre. On avait besoin d'infirmières ; et de quelques espionnes comme le montre l'autobiographie de l'aviatrice Marthe Richard recrutée par les services de renseignements français, non pas pour voler mais pour séduire un officier allemand.

Ce n'est donc qu'à la fin des hostilités que les femmes purent reprendre le chemin des aérodromes. Le nombre des brevetées augmenta rapidement, mais contrairement aux aviateurs qui passèrent sans transition de l'armée de l'air aux premières lignes commerciales qui se créaient un peu partout en France, les femmes continuèrent à être tenues à l'écart. Pour les aviatrices, les débouchés étaient rares et 
l'on s'attendait à ce que les femmes se cantonnent dans les rôles de passagères et d'observatrices, comme ce fut le cas de l'écrivaine et journaliste Lise Faure-Favier.

Humaniste et grande voyageuse, Faure-Favier n'apprit jamais à piloter mais elle se passionna pour l'aéronautique, devint l'amie de nombreux aviateurs et publia d'innombrables articles sur le sujet, plusieurs romans et de nombreux Guides de voyages aériens. Bien qu'il n'ait pas pour thème principal l'aviation, son roman Blanche et noir publié en 1928 est pertinent car il évoque l'Afrique, imagine un pilote noir sénégalais rejoignant une escadrille de l'armée française pendant la grande guerre et dénonce avec talent le sexisme, le racisme et l'ambiance étouffante de la petite bourgeoisie de province qui rendaient si difficiles les projets d'émancipation des jeunes femmes des années vingt. Une page certainement inspirée de l'expérience de l'auteure, évoque la naissance de la passion de son héroïne pour l'aviation :

En mars 1919, à nouveau les hasards du journalisme firent de Jeanne Rieux (c'était désormais mon nom d'écrivain) un reporter aérien. Les hasards du journalisme, mais surtout un goût romantique pour les voyages et les aventures que je n'avais pas encore pu satisfaire, me firent prendre place dans le premier avion civil pour le premier voyage aérien. [...] le 18 juillet, je faisais dans un avion de sport, le double voyage Paris-Londres, et j'en rapportais le soir même le récit des fêtes de la victoire, très fière d'être ainsi la première femme ayant survolé deux fois la Manche. Depuis, que de voyages, combien d'orages, mais en retour, que de reportages, que d'ouvrages ! (114)

En lisant ce passage, on ne peut s'empêcher de penser à la romancière Colette qui, en 1919, embarqua elle aussi avec sept autres journalistes dans un des premiers vols pour Londres, malheureusement détourné sur Bruxelles suite au refus des Britanniques de laisser l'avion atterrir en Angleterre. Etait-ce un signe avant-coureur des luttes d'influence qu'allaient se livrer les compagnies d'aviation nationales par la suite ? Qui sait. Mais pour Lise Faure-Favier, la conquête des airs était au-dessus des conflits d'intérêts et des nationalismes exacerbés qui avaient précipité le monde dans l'horreur quelques années auparavant. L'aviation représentait pour elle la liberté et une «fraternelle réalité » entre les peuples, comme en témoigne la fin du roman qui s'achève par une envolée lyrique de la narratrice sur le point de s'élancer vers Dakar : «Fraternité des races, je vole vers vous, s'exclame-t-elle, avant de déploye[r] la carte où un trait rectiligne marquait le trajet aérien des Cévennes à Dakar, semblable à celui que [s]on rêve d'enfant traçait jadis sur la mappemonde : Cap au sud !» (152) 


\section{Survoler l'Afrique}

Les progrès technologiques qui marquent les années vingt permettent le développement d'un réseau complexe de lignes aériennes transportant courrier et passagers un peu partout dans le monde. Malheureusement, la décision d'autoriser les femmes sur les lignes commerciales en 1926 ne change rien en pratique et les aviatrices continuent à être maintenues à l'écart de l'aviation commerciale. Il y pourtant quelques exceptions hors de l'Hexagone : l'une d'entre elles, la Kenyane d'origine anglaise Beryl Markham qui devint pilote professionnelle au Kenya après avoir abandonné un élevage de purs sang. Son autobiographie Vers l'ouest avec la nuit évoque sa décision d'apprendre à piloter au début des années trente, son apprentissage à Nairobi sur un D.H. Gipsy Moth avec Tom Black et la réussite de son brevet $\mathrm{B}$ après mille heures de vol et un examen théorique de navigation basé sur des livres «dont les auteurs semblaient ne pouvoir s'exprimer qu'avec des mots d'une longueur démesurée »(219). Une fois son brevet professionnel en poche, Beryl Markham acheta un avion et commença à gagner sa vie comme pilote :

Avant que Tom ne quitte la Wilson Airways pour devenir le pilote de Lord Furness (et plus tard du Prince de Galles), dit-elle, nous nous rencontrions régulièrement le soir pour prendre l'apéritif ou dîner ensemble et pour parler de nos vols et de mille autres choses. Je travaillais alors comme pilote indépendant, transportant du courrier, des passagers, des provisions pour les safaris, ou toute autre cargaison. [...] Nous quittions souvent l'aéroport de Nairobi juste après l'aube Tom en route pour l'Abyssinie, et moi pour le Soudan anglo-égyptien, le Tanganyika, la Rhodésie du Nord, ou n'importe quelle autre destination où m'appelait un contrat (220).

Beryl Markham est l'une des rares femmes de l'époque à avoir réussi à vivre de son métier de pilote professionnelle, mais cela n'empêcha pas de nombreuses femmes de continuer à voler pour le plaisir. Certaines trouvèrent des sponsors et réalisèrent des exploits qui les rendirent célèbres, comme l'Allemande Elly Beinhorn qui rallia la Guinée-Bissau depuis l'Allemagne en 1931 (et mourut dans son lit à l'âge de cent ans en 2007). Elle fut contrainte à un atterrissage forcé en plein Sahara sur le chemin du retour et fut secourue par une caravane qui lui permit d'arriver à Tombouctou, épuisée et malade, après plusieurs jours de marche. Loin d'être découragée par sa mésaventure, Elly Beinhorn s'envola l'année suivante en direction de l'Asie puis de l'Australie où elle arriva en 1932. 
Un ouvrage de Maurice Martin du Gard publié à la fin de 1931 mentionne le passage d'une aviatrice allemande à Bamako, et bien que ni le nom de cette personne ni la date de cette rencontre ne soit précisé par Martin du Gard, on peut facilement imaginer que son compte-rendu illustre assez bien la réception chaleureuse réservée à Elly Beinhorn et de manière générale aux aviatrices de toutes nationalités dont la présence éphémère créait l'événement, au cœur de l'Afrique comme ailleurs :

A peine y avait-il quelques heures qu'une avionnette avait demandé le terrain et atterri comme une fleur. Une jolie petite personne en était descendue, avec sa boîte à poudre, l'air le plus modeste, une robe toute simple et courte, des bas de sport qui laissaient voir ses genoux. Elle arrivait comme ça toute seule, de Berlin, ayant traversé l'Allemagne, la France, l'Espagne et l'Afrique, au long de la Mauritanie, puis de la Guinée portugaise, sa dernière escale, ayant souffert des différences de température incroyable et s'étant crue perdue plus d'une fois. Nos officiers firent fête à l'audacieuse. Et comme il lui fallait obtenir une autorisation pour continuer son voyage vers Tombouctou, on l'emmena au gouverneur qui la reteint à déjeuner. [...] Nous étions tous fort empressés autour d'elle à admirer cette simplicité et cette gentillesse, après un tel exploit. (123-124)

Bien d'autres femmes pilotes s'illustrèrent pendant l'entre-deux-guerres. Certaines, comme Maryse Hilsz, menèrent leur carrière à un train d'enfer. En deux ans Hilsz accumula deux cents heures de vol, mille cinq cents baptêmes de l'air, elle participa à une quarantaine de meetings aériens et en 1932, elle vola de Paris à Madagascar au cours d'un long périple africain commandité par le Ministre de l'air (Nicolaou et Mismes-Thomas 118-119).

Ce sont les noms de ces femmes d'exception que l'Histoire a retenu, mais des milliers d'autres restées anonymes ont continué à s'élancer dans les airs en quête de satisfactions plus personnelles - et souvent à peine moins hasardeuses. Le témoignage de la Comtesse de Forges publié sous le titre de Sur les ailes de mon fils est intéressant à cet égard car il montre qu'en marge de l'aviation-spectacle qui captive les foules et de l'aviation commerciale qui s'implante un peu partout, on assiste aussi aux débuts d'une aviation de loisir qui commence à donner une apparence de normalité à une activité considérée jusque-là comme extraordinaire.

\section{Début du tourisme aérien}

En contraste avec les jeunes femmes intrépides et déterminées à braver le qu'en-dira-t-on pour réaliser leur rêve, la Comtesse de Forges a déjà un certain âge 
lorsqu'elle commence à s'intéresser à l'aviation, non pas parce que l'idée de conquérir les cieux la tente mais parce que son fils passionné par les avions devient pilote et invite sa mère à l'accompagner pour admirer le monde d'en haut. Le voyage aérien qui la conduit à survoler l'Afrique, n'a donc d'autre but que de passer de bons moments avec son fils : «Sans doute aurais-je ignoré l'attirance de l'espace, écrit-elle en exergue de son ouvrage, si tu ne l'avais toi-même subie, mais tu as voulu des ailes et il m'a paru tout simple de m'envoler à tes côtés » (8).

Les premières pages du roman réitèrent ce sentiment de bonheur, le plaisir d'une mère de découvrir le monde «sur les ailes » de son fils bien aimé :

Une course trépidante, accélérée, et puis plus rien : l'impression de glisser dans le rêve...

J'ai regardé en bas : le terrain m'est apparu comme un mouchoir. [...] J'ai songé : pour que l'Aiglon ait pu monter si vite, il faut vraiment que la joie soit une cargaison légère! Joie de partir, joie d'aller vers le soleil, joie d'avoir mon fils pour pilote... comment toute cette joie a-telle pu entrer sans l'alourdir dans notre étroite carlingue ?

Et j'ai souri en pensant à la recommandation de Paul : «Surtout, maman, pas trop de bagages ! Tu peux emporter ta brosse à dents, mais fais lui mettre un manche démontable !»Certes, il n'est pas grand notre avion! Déguisée en Esquimau et mon parachute sur le dos, j'ai eu quelque peine à m'introduire dans l'habitacle réservé au passager, à l'avant du fuselage.

Mais, peu à peu, j’y ai fait mon nid, tout s'est tassé. Je me sens chez moi. (10)

Ecrit d'une plume alerte, la relation de voyage de la Comtesse et de son fils Paul de Forges nous entraîne dans un périple qui conduit la narratrice de Nevers à Tombouctou en passant par la Lyon, Marseille, Bastia, Cagliari, Tunis, Alger, Oran, Colomb Béchar, Reggan, Bidon 5 Aguelock et Gao. Voyageant «léger», comme les jeunes qui partiront à l'aventure une génération plus tard, la Comtesse découvre un monde nouveau qui se déroule sous ses yeux comme un «grand livre d'images ». Elle passe sans transition d'un ciel bleu azur aux conditions atmosphériques les plus exécrables et chaque escale donne lieu à des surprises, des émerveillements, des rencontres inattendues : ici, c'est un patron d'hôtel serviable et loquace ; là, le départ des pèlerins pour la Mecque ; et à Gao c'est le Simoun, «l'oiseau rouge » (98) de Saint-Exupéry que son mécanicien Prévot est en train de ravitailler lorsqu'ils se posent sur l'aire brûlante du petit aéroport. Puis c'est la découverte du fleuve Niger, une promenade en pirogue «qui glisse nonchalamment sur le lac-fleuve » (111) et enfin Tombouctou à la longue l'histoire : «on n'aborde pas sans quelque émoi, dit- 
elle, une ville qui pendant plus de sept cents ans, est restée inaccessible aux Européens » (120).

La seconde guerre mondiale, déclarée l'année de la publication du livre de la Comtesse de Forges, n'empêche ni les avions de voler ni le matériel aérien de progresser à pas de géant, mais le début des hostilités va par contre briser l'élan d'un tourisme aérien naissant, tout comme il va réduire à la portion congrue les aspirations des femmes pilotes, du moins en France où, comme le suggère Bernard Marck, «pas plus le rêve d'une aviation sanitaire caressé par Marie Marvingt et Hélène Dutrieu, que celui d'une escadrille composée d'aviatrices, souhaitée par Marthe Richard, ne déboucheront sur la réalité, du moins en France » (262). Tout au plus, quatre pilotes dont Maryse Hilsz, obtiennent-elle une dérogation par décret en 1939. La création des pilotes auxiliaires féminines de l'air en 1940 n'ouvre qu'un nombre très limité de postes à cause des innombrables restrictions imposées aux candidates : «En 1939, comme mille ans auparavant, les états-majors jugent toujours inopportune la présence des femmes sur le champ de bataille. La guerre, bientôt perdue, ne modifie pas les mentalités » (263).

\section{Les 'as' n'étaient pas françaises}

D'autres pays adoptèrent une approche plus flexible. En Angleterre, Pauline Gower créa la section féminine de l'Air Transport Auxiliary qui convoya des milliers d'avions depuis les usines jusqu'aux bases aériennes. En Union soviétique, quelques six-cents aviatrices servant dans trois unités féminines, prirent part aux bombardements et aux engagements directs avec l'ennemi. La plus célèbre d'entre elle fut peut-être La Rose de Stalingrad, Lily Litvak, la pilote de chasse aux douze victoires dont la vie est évoquée dans le roman de Valérie Bénaïm et Jean-Claude Hallé. Ou encore l'Allemande Hanna Reitsch qui fut détachée au centre d'essais en vol de la Luftwaffe, devint pilote d'essai pendant la guerre et résida au Ghana de 1962 à 1966, un séjour suite auquel elle écrivit l'ouvrage J'ai volé pour Kwame Nkrumah. On retrouve aussi la trace d'une de ces pilotes de guerre allemandes réfugiées en Afrique dans l'ouvrage-reportage de la journaliste française Christine Garnier qui séjourna brièvement au Libéria à la fin des années quarante. Elle y rencontra dans l'hôtel de Monrovia où elle était descendue, écrit-elle, une jeune Allemande dont elle n'apprit pas grand chose si ce n'est que «certains prétendaient qu'elle avait été une aviatrice célèbre, et qu'elle avait effectué des vol d'essai pour Messerschmidt pendant 
la guerre »(129). Cette allusion à une femme pilote oubliée par l'histoire est symptomatique des lacunes entourant la contribution des femmes au développement de l'aviation en temps de guerre comme en temps de paix.

\section{Le métier de pilote de ligne reste une chasse gardée des hommes}

Au sortir de la guerre, le réseau autonome d'Air Afrique et la compagnie Air France (fondée en 1933 pour regrouper les cinq compagnies françaises indépendantes qui se disputaient la faveur des premiers usagers) prirent un essor considérable. L'aviation moderne était née mais cela ne signifiait pas pour autant que l'accès au métier de pilote de ligne fût plus ouvert aux femmes qu'avant-guerre. Comme le suggère un article de Michel Léveillard consacré à la pilote Monique Caillard, quelles que soient leurs qualités et prédispositions pour l'aviation, les femmes étaient vilipendées lorsqu'elles prétendaient empiéter sur des prérogatives masculines :

Plusieurs jeunes femmes fréquentaient les Aéro-Clubs à mon époque [les années cinquante], mais je dois avouer en toute franchise que très peu de mes «confrères » les prenaient au sérieux, et ces jeunes femmes qui devaient quelques fois même supporter d'être ridiculisées et découragées abandonnaient peut-être trop facilement leur rêve de devenir pilote. (np)

Abandonner ne faisait pas partie du vocabulaire de Monique Caillard, une institutrice de vingt-trois ans contaminée par le virus de l'aviation après avoir assisté à un meeting aérien en 1949. Douée d'un talent particulier pour le pilotage, elle passa plusieurs brevets, devient monitrice à l'Aéro-Club de Normandie, participa à des stages de voltige puis à de nombreux meetings aériens et finit par obtenir le «grand » diplôme, dit Léveillard, celui qui couronne la carrière des pilotes : le Brevet de pilote de transport public qui permettait de devenir pilote de ligne. Ce parcours sans faute et l'obtention de tous les brevets requis ne permirent cependant pas à Monique Caillard de rejoindre les rangs des pilotes de ligne. Comme le dit Léveillard : "C'était un rêve, et seulement un rêve pour une femme à cette époque. Il faudra attendre beaucoup d'années à ces femmes, pour pénétrer ce domaine et pourtant Monique avait toutes les qualifications, et elle aurait bien mérité cette belle profession » (np).

Faute de pouvoir devenir pilote de ligne, Monique Caillard accepta alors un emploi en Afrique noire comme pilote agricole chargée de vaporiser de l'insecticide sur les sauterelles. Elle allait rester huit ans en Afrique. 


\section{Enfin une femme dans le cockpit}

Il faudra attendre 1974 pour qu'une femme, Danielle Décuré, soit engagée comme pilote de ligne chez Air France. Le parcours du combattant de cette pionnière au caractère pugnace est décrit dans son autobiographie, un livre plein de verve et d'humour intitulé Vous avez-vu le pilote ? C'est une femme. La lecture de cet ouvrage est un régal et quelques pages relatant les péripéties d'un vol au Togo au début de 1974 donnent le ton :

On est à l'automne 1973. Les Egyptiens profitent du Kippour pour aller jouer la guerre avec les Israéliens. La guerre du pétrole commence. [...] le prix du pétrole sur les aéroports change chaque jour, les compagnies pétrolières n'acceptent plus les cartes de crédit, il faut emporter des tas d'argent liquide pour payer cash le kérosène. [...] En janvier 1974, je pars pour une courte semaine au Togo. J'ai pris mes précautions et emporté une sacrée réserve. Première escale : Tamanrasset. Le pompiste accepte ma carte de crédit. Choukrane! Tamanrasset - Lomé, le vent pousse. Un peu de repos : le marché où on marchande des boubous africains typiques made in Japan, les cocotiers le long de la mer, les lagunes du Dahomey tout proche, les gros lézards verts qui viennent nous regarder de travers parce que nous leur faisons de la concurrence à lézarder à plat ventre au bord de la piscine. Il faut rentrer vers le froid parisien. Lomé - Niamey où je paie cash le kérosène pour aller à Tamanrasset. [...] A Tam, catastrophe, le pompiste veut bien nous faire le plein, à condition que nous payions comptant et que nous payions aussi le plein que nous avions fait quelques jours avant. [...] J'avais payé en argent liquide les frais d'hôtel, [...] les taxes d'atterrissage et je n'avais plus assez d'argent pour régler les deux factures. J'ai proposé de faire la plonge, ça n'a pas fait rire. J'ai donc fait mes fonds de poche, ceux de l'hôtesse et ceux du copilote; j'ai fait la quête auprès de mes passagers qui, naturellement, n'étaient pas des gens fauchés. Nous sommes arrivés à Paris à treize, avec un jet privé et douze francs cinquante au total. En plus de ce souvenir, j'ai ramené dans mon ventre un virus inconnu et incongru qui a failli dans un premier temps m'envoyer finir mes jours au Paradis. (131-134)

Comme Danielle Décuré et toutes les femmes nées avant guerre, Anne Spoerry connut «la malédiction d'être née femme» (Décuré 7) quand on voulait exercer un métier réservé aux hommes. La destinée d'Anne Spoerry fut exceptionnelle mais avant de s'intéresser à l'aviation, sur le tard, c'est son désir de devenir médecin au Kenya, au tout début des années cinquante, qui lui vaut l'animosité des uns et l'incompréhension des autres. Le chef des services médicaux au ministère de la santé à Nairobi l'ayant invitée à poser sa candidature pour un poste dans le district de Marsabit, elle envoie son dossier au commissaire de district qui ne 
fait qu'un bond : «Une femme médecin, jamais. Et pas mariée en plus » (Spoerry 85). Une nouvelle postulation à Naivasha aboutit au même résultat : «Là non plus on ne voulait pas d'une femme » (86). Ce n'est qu'à sa troisième tentative qu'elle put enfin s'installer dans un petit village où elle allait rester quatorze ans. En 1963, déprimée par le tour que prenaient les évènements politiques, elle décida d'apprendre à voler pour se changer les idées et « acquérir un nouvel espace de liberté » (128).

\section{L'aviation sanitaire}

Le souvenir de Beryl Marham était toujours présent dans les mémoires, ditelle, et la rencontre d'Anne Spoerry avec l'aviatrice June Sutherland fut déterminant comme elle le raconte dans son autobiographie On m'appelle Mama Daktari :

Je connaissais [...] June Sutherland, qui avait été décorée par la Reine pour son action pendant la guerre du Congo. Avec son avion, elle allait se poser sur des terrains de fortune, en pleine zone de combat, et elle évacuait les femmes et les enfants en détresse vers le Rwanda. Elle en a sauvé beaucoup. C'est elle qui m'a emmenée pour la première fois à Loyangalani et au lac Turkana, en 1961. Le vol a été magnifique, et je crois bien que c'est à ce moment-là que j'ai pensé sérieusement à piloter moi-même. (135)

L'histoire ne s'arrête pas là, car à l'aube de la cinquantaine, Anne Spoerry rejoint les rangs de l'African Medical Research Foundation comme médecin volant et, surnommée Mama Daktari par ses patients et son entourage, elle poursuit ses activités avec cette association jusqu'à l'âge de quatre-vingts ans, survolant inlassablement le nord-est du Kenya pour gagner les endroits les plus isolés et soigner, immuniser et informer ses patients sur les maladies infectieuses et la contraception.

Cette destinée ne va pas sans rappeler celle de Valérie André, elle aussi une jeune femme dynamique et déterminée qui est engagée comme médecin-capitaine en 1949 en Indochine. Bravant les réticences de sa hiérarchie, elle devient pilote d'hélicoptère et se voit confier par la suite un grand nombre d'évacuations médicales héliportées. Affectée en Algérie en 1959, elle y poursuit le même genre d'activité et entreprend plusieurs centaines d'évacuations en territoire algérien avant de rentrer en France où elle se hisse au sommet de la hiérarchie militaire. Deux ouvrages de Valérie André évoquent ce parcours de vie hors du commun : Ici, Ventilateur! Extraits d'un carnet de vol et Madame le général. 
Si apprendre à voler est plus facile de nos jours que dans le passé, les mêmes clivages existent encore lorsqu'il s'agit de progresser dans la hiérarchie des compagnies aériennes lorsqu'on est une femme : même s'il est en augmentation, seul un très petit pourcentage de femmes sont aux commandes des avions d'Air France aujourd'hui encore, et la situation est la même en Afrique où les femmes pilotes font figure d'exception.

\section{Pilotes africaines}

Dans ce domaine, au Nord et plus encore au Sud, les choses n'ont progressé que très lentement depuis l'époque où Bessie Coleman, jeune Américaine d'origine africaine, arriva en France pour apprendre à voler, ce qui lui était interdit dans son pays parce qu'elle était noire. Elle devint la première aviatrice noire en 1921. Combien d'Africaines ont-elles sillonné les cieux depuis aux commandes d'un avion petit ou grand, est matière à conjecture, mais la photo pleine page de Thérèse Bella Mbida (née en 1932) aux commandes de son Cessna, proposée dans un ouvrage intitulé Hommage à la femme camerounaise (Okani 40), montre que les Africaines n'ont pas attendu le vingt-et-unième siècle pour s'intéresser à l'aviation. Près d'un siècle après Bessie Coleman, plusieurs Africaines peuvent servir d'exemples aux jeunes filles qui rêvent aujourd'hui de devenir pilote de ligne, comme Paméla Améga, une élève de seize ans du lycée Tokoin Saint Joseph de Lomé qui, dit un petit article lui étant consacré dans la presse locale, «manie le manche de son Cessna T152 avec dextérité. [...] en attendant de s'installer un jour dans le cockpit d'un Boeing » (Et voici, np.).

Il existe en Afrique comme partout des familles progressives qui encouragent leur filles à aller au bout de leurs ambitions, mais il y en a aussi beaucoup qui restent attachées au statu quo comme le montrent Tante Fatoumata et sa jeune nièce Shadé, deux personnages imaginés par Anne Laflaquière dans un ouvrage pour les jeunes :

- Te voici bonne à marier, ma nièce.

- Merci bien ! Je veux d'abord mon diplôme d'aviateur !

- Aviateur ! Quelle horreur !... Un bon mari, voilà ce qu'il te faut !

- Ma mère prit ma défense (30-31).

$[\ldots]$

- Viendras-tu me voir au Mali ?

- Dès que je serai pilote de ligne, Tantie.

Elle grogna :

- Ta, ta, ta !... Je te trouverai un bon mari, petite mule ! un gars sérieux, vaillant et pieux, qui te fera oublier tes avions ! (54) 
Toutefois, comme le dit la romancière Gisèle Hountondji, « depuis longtemps, bien des femmes ont compris que 'leur premier mari, c'est leur métier' » (2). Dès lors, maintes jeunes filles trouvent leur vocation avant «le gars sérieux » qui leur fera tourner la tête. Touria Chaoui en fournit la preuve. Echappant au ségrégationnisme de son milieu et du Protectorat français du Maroc qui ne concevaient pas qu'une jeune Marocaine puisse devenir pilote, elle obtint son brevet en 1952, à l'âge de seize ans et devint ainsi la première aviatrice marocaine - et du monde arabe. Son assassinat en 1956, alors qu'elle n'avait pas vingt ans, mit un terme prématuré aux premiers succès aériens féminins d'Afrique du Nord. Cela ne les interrompit cependant pas définitivement car en 1986, Bouchra Bernoussi et Oumaima Sayeh, deux compatriotes de Touria Chaoui, devenaient les premières pilotes de ligne marocaines.

Au Congo, la première femme pilote du pays s'appelle Adine Ossebi Ndiaye. D'abord copilote à Air Afrique pendant cinq ans, elle devient copilote à Air Sénégal international après la faillite d'Air Afrique, puis copilote à Royal Air Maroc lorsque Air Sénégal dépose à son tour son bilan. S'il est difficile de devenir pilote, il n'est pas facile non plus de le rester.

\section{Une nouvelle génération aux commandes}

La littérature africaine d'expression française nous a habitués aux femmes prenant l'avion parmi les passagers pour se rendre à Paris ou ailleurs. Les femmes voyageant dans le cockpit sont plutôt rares. Toutefois, un roman d'Assamala Amoi publié en 2010 reflète les espoirs d'une nouvelle génération qui n'attend que le moment d'abandonner au passé les écueils du népotisme, du chômage et des anciens clivages pour passer aux commandes.

Avion par terre raconte l'histoire d'Alizéta, une jeune femme qui réalise un rêve d'enfant en devenant pilote puis Commandant de bord dans une petite compagnie africaine. C'est l'un des tous premiers romans africains évoquant la vie d'une pilote africaine contemporaine aux prises avec les vicissitudes de son époque. A ce titre, cet ouvrage est important, mais son intérêt ne s'arrête pas là. A travers le parcours sans faute d'Alizéta on découvre les préoccupations sociales de la jeune femme, son humanité, sa recherche du bonheur. On est témoin des abus et des passedroits qui conduisent inexorablement la compagnie qui l'emploie à la ruine. On partage l'incrédulité de la narratrice face «à la gestion calamiteuse » (406), 
l'incompétence et la cupidité de ceux qui ont envoyé l'African Skylines - alias Air Afrique - «Dans le cimetière des avions par terre / Qui ne décolleront plus jamais » (412).

Mais dans le même temps, on assiste aussi à la naissance de Lazarus Lines, une nouvelle compagnie aérienne africaine moderne, souple et disciplinée. Une entreprise ouverte aux femmes pilotes qui met l'accent sur la fiabilité, la transparence, la satisfaction des voyageurs et le recrutement d'employés responsables. Les turbulences qui ont cloué au sol les avions de l'African Skylines, ne viennent pas du ciel mais des mains des hommes, dit la narratrice, et c'est un changement d'attitude face aux autoroutes du savoir, au commerce international et à la mondialisation (412) qui ouvrent de nouvelles perspectives d'avenir symbolisées par la trajectoire de la capitaine Alizéa Ba.

Les raisons qui poussent Alizéa à s'intéresser à l'aviation sont également intéressantes et elles dépassent la singularité d'une hérö̈ne de roman. Elles soulignent l'importance de l'éducation des enfants dans les choix qu'ils feront plus tard. En ce qui concerne Alizéa, c'est une excursion à l'aéroport organisée par l'école Bessie Coleman où elle est élève de cours élémentaire, qui détermine le cours que va prendre sa vie. Elle a neuf ans et le commandant de bord qui les accueille est une femme d'une trentaine d'années enjouée et sympathique que la petite fille ne quitte plus des yeux. Et la fascination d'Alizéa pour «les signaux et les voyants lumineux [qui] l'attirent comme des aimants » (24) lors de sa visite du cockpit, deviendra le fil rouge qui guidera sa vie. Comme le relève la narratrice après que la commandante Dos Anjos a posé cérémonieusement sa casquette sur la tête d'Alizéa :

La pilote reconnut dans les yeux de l'enfant la flamme qui brûlait dans [le regard] de tous ceux que dévorait la passion de voler, le désir d'aller à l'assaut de l'infini, de mesurer ses rêves avec le fil de l'infini. C'était un feu sacré qui réchauffait mais ne consumait pas ni ne réduisait en cendres $(25)$.

Dès le début de l'aviation, des femmes de toutes origines ont ressenti cet impérieux besoin de mesurer leurs rêves à l'aune de l'infini, d'être libres. De la baronne de Laroche à la commandante de bord Alizéa Ba imaginée par Assamala Amoi dans son dernier roman, plusieurs générations d'aviatrices ont bravé les préjugés pour s'élancer dans les airs. De nombreux ouvrages y attestent leur présence dès la première heure et ces témoignages méritent une place de choix dans l'univers littéraire du vingtième et unième siècle. 


\section{Bibliographie}

Amoi, Assamala. Avion par terre. Paris : Anibwe, 2010.

André, Valérie. Ici, Ventilateur! Extraits d'un carnet de vol. Paris : Calmann-Lévy, 1954.

---. Madame le général. Paris : Perrin, 1988.

Auriol, Jacqueline. Vivre et voler. Paris : Flammarion, 1968.

Béal, Jacques. Bessie Coleman. L'ange noir. Paris : Michalon, 2008.

Bébaïm, Valérie et Jean-Claude Hallé. La rose de Stalingrad. Paris : Flammarion, 2005.

Beinhorn, Elly. Alleinflug. Mein Leben. Munich : Langen-Müller, 1977.

Chambe, René. Histoire de l'aviation des origines à nos jours. Paris : Flammarion, 1958.

Chazeaux, Olivier de. Maryse Hilsz, la femme qui aimait tant le ciel. Paris : Lattès, 1999.

Décuré, Danielle. Vous avez-vu le pilote? C'est une femme. Paris : Laffont, 1982.

«Et voici la plus jeune pilote togolaise». Togo. 2010. Web 1 jan 2012. $<$ http://www.republicoftogo.com/Toutes-les-rubriques/Societe/Et-voici-laplus-jeune-pilote-togolaise>.

Faure-Favier, Lise. Blanche et noir. 1928. Paris : L'Harmattan, 2006.

Forges, Comtesse de. Sur les ailes de mon fils. A Tombouctou par le Tanezrouft. Paris : Grasset, 1939.

Garnier, Christine. Les héros sont fatigués. Paris : Grasset, 1953.

Hountondji, Gisèle. «Les bêtises de Napoléon ». La Nouvelle Tribune (Cotonou). 118, 8 mai 2002, 2. Web 1 jan 2012. <http://aflit.arts.uwa.edu.au/Hountondji_chronique1.html>.

Laflaquière, Anne. Fatoumata, ma tante. Paris : L'Harmattan, 1990.

Léveillard, Michel. «Monique Caillard : 'The right stuff' au féminin ». Aérostories, 2001. Web. 1 janv 2012. < http://aerostories.free.fr/pilotes/france/caillard >.

Marck, Bernard. Les aviatrices. Des pionnières aux cosmonautes. Paris : L'Archipel, 1993.

Markham, Beryl [1942]. Vers l'ouest avec la nuit. Tr. Viviane Markham. Paris : Castor Poche Flammarion, 1995.

Martin du Gard, Maurice. Courrier d'Afrique. Sénégal - Soudan - Guinée. Paris : Flammarion, 1931. 
Nicolaou, Stéphane, et Elizabeth Mismes-Thomas. Aviatrices. Un siècle d'aviation féminine française. Levallois-Perret : Altipresse, 2004.

Okani, Rachel Claire. Hommage à la femme camerounaise. Yaoundé : CIAG, 1995.

Reitsch, Hanna. Ich flog für Kwame Nkrumah. Munich : Lehmanns, 1968.

Richard, Marthe. Mon destin de femme. Paris : Laffont, 1974.

Saint-Exupéry, Antoine de. Courrier Sud. Paris : Gallimard, 1929.

Spoerry, Anne. On m'appelle Mama Daktari. Paris : Lattès, 1994.

\begin{abstract}
Note
${ }^{1} \mathrm{Si}$ la baronne de Laroche est la première femme pilote brevetée, c'est Thérèse Peletier qui fut la première femme à avoir pris les commandes d'un avion en 1908. A noter qu'à l'ère des ballons et des dirigeables, de très nombreuses femmes eurent l'occasion de regarder le monde d'en-haut. Elisabeth Thible, qui sauta dans la nacelle d'une des toutes premières montgolfières au moment où celle-ci s'arrachait du sol, devint en 1784, à Lyon, la première femme embarquant dans un aéronef ; la marquise de Montalembert, la comtesse du même nom, la comtesse de Podenas et mademoiselle de Lagarde s'élevèrent de quelques centaines de mètres au-dessus de Paris à bord d'un ballon captif en 1794 avec Jean-François Pilâtre du Rozier ; et par la suite, de très nombreuses femmes se passionnèrent pour les vols en ballons.
\end{abstract}

